\title{
Microvascular Study of the Retrolaminar Optic Nerve in Man: the Possible Significance in Anterior Ischaemic Optic Neuropathy
}

\author{
J. M. OLVER, ${ }^{1,2}$ D. J. SPALTON,${ }^{3}$ A. C. E. McCARTNEY ${ }^{2}$ \\ London
}

\begin{abstract}
Summary
The morphology of the circle of Haller and Zinn and its variations were examined using methyl-methacrylate microvascular corrosion casting of human orbits obtained at post-mortem. It was found to be an elliptical microvascular anastomosis formed by branches of the medial and lateral para-optic short posterior ciliary arteries. The ellipse was divided into superior and inferior parts by the entry points of these branches into the eye, providing an altitudinal blood supply to the retrolaminar optic nerve. Morphological variations in terms of form, position and branches existed between subjects and between eyes from the same subject. The clinical implications of an elliptical 'circle' of Haller and Zinn providing an altitudinal blood supply to the retrolaminar optic nerve are relevant to the pathogenesis of altitudinal visual field defects in anterior ischaemic optic neuropathy.
\end{abstract}

Anterior ischaemic optic neuropathy (AION) exists in two forms, arteritic (associated with giant cell arteritis) and non-arteritic (idiopathic). Non-arteritic anterior ischaemic opticneuropathy is a relatively common cause of loss of vision in middle age. ${ }^{1,2}$ It is characterised by altitudinal patterns of visual field loss in which the inferior field is preferentially affected $^{1-3}$ (most commonly infero-nasal) associated with characteristic asymmetrical pallid optic disc oedema. The few cases of anterior ischaemic optic neuropathy that have been examined histopathologically locate the infarction to the posterior laminar and retrolaminar optic nerve. ${ }^{4,5}$

Most authors suggest this region has a blood supply from pial branches from the medial and lateral bundles of the short pos- terior ciliary arteries and recurrent choroidal branches. ${ }^{6-8}$ However, it is difficult to explain the altitudinal nature of visual field defects on such a purely horizontal vascular supply. These defects have previously been attributed to watershed infarctions.

We have applied the technique of methyl methacrylate microvascular corrosion casting to elucidate the microvasculature of the laminar and retrolaminar optic nerve in man in order to try and answer two clinically relevant questions related to anterior ischaemic optic neuropathy: (i) Why is the field defect most commonly altitudinal and does this reflect an altitudinal blood supply to the retrolaminar optic nerve? (ii) Why is there preferentially a lower visual field loss, and is this also anatomically determined? 


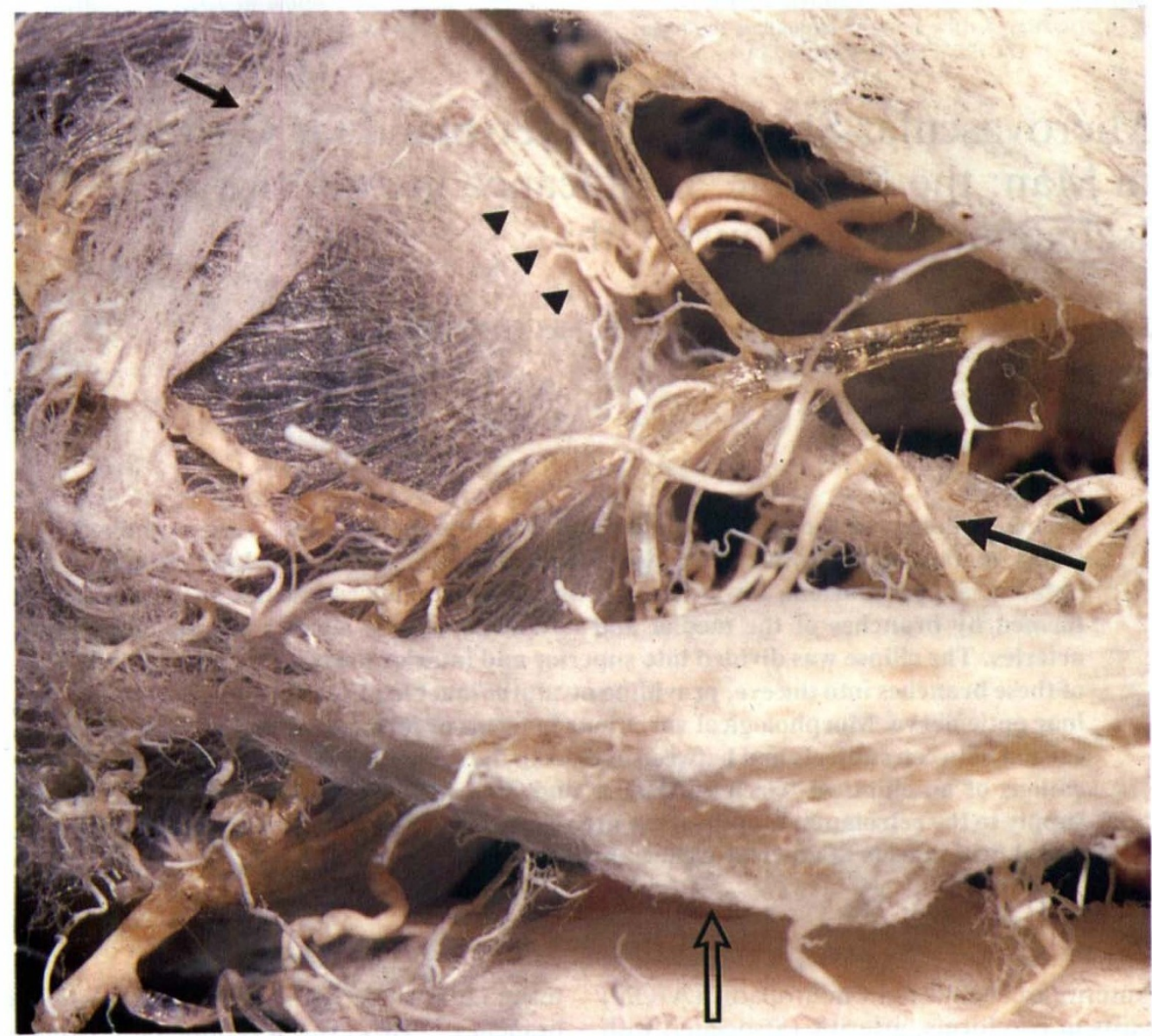

Fig. 1. Macroscopic appearance of vascular cast of the posterior globe and orbital vessels showing the optic nerve (long solid arrow), short posterior ciliary arteries over the macula (3 small arrowheads), inferior oblique muscle close to insertion (short solid arrows) and inferior rectus (empty arrow).

\section{Methods}

Human cadaver orbits obtained $36-48$ hours post-mortem were prepared for micro-vascular corrosion casting and injected via the ophthalmic artery with thinned Batson's mixture (methyl methacrylate based) according to our standard technique. ${ }^{9}$ Following corrosion in six Molar potassium hydroxide the casts were washed in distilled water and air dried. The optic nerve, rectus muscles and posterior ciliary vessels were then divided approximately one centimetre behind the globe to separate the globe and retrolaminar optic nerve cast intact from the orbital vasculature. The casts were examined macroscopically under a binocular dissecting microscope to identify the short posterior ciliary bundles, circle of Haller and Zinn if visible and to orientate the eye. The optic nerve and short posterior ciliary arteries were then further trimmed to within $1.5 \mathrm{~mm}$ of the posterior aspect of the cast. The casts were sputter coated with $50 \mathrm{~nm}$ gold and examined by scanning electron microscopy either whole or as dissected fragments of posterior pole and retrolaminar optic nerve mounted on an aluminium stub. Scanning electron photomicrographs were taken at $\times 25$ and $\times 60$ magnification at a tilt of $0^{\circ}, 45^{\circ}$ and $90^{\circ}$ (ie side on, obliquely and from above the optic nerve).

\section{Results}

Vascular casts from 21 adult eyes from 17 cadavers (ages 21-89 yrs) were examined 


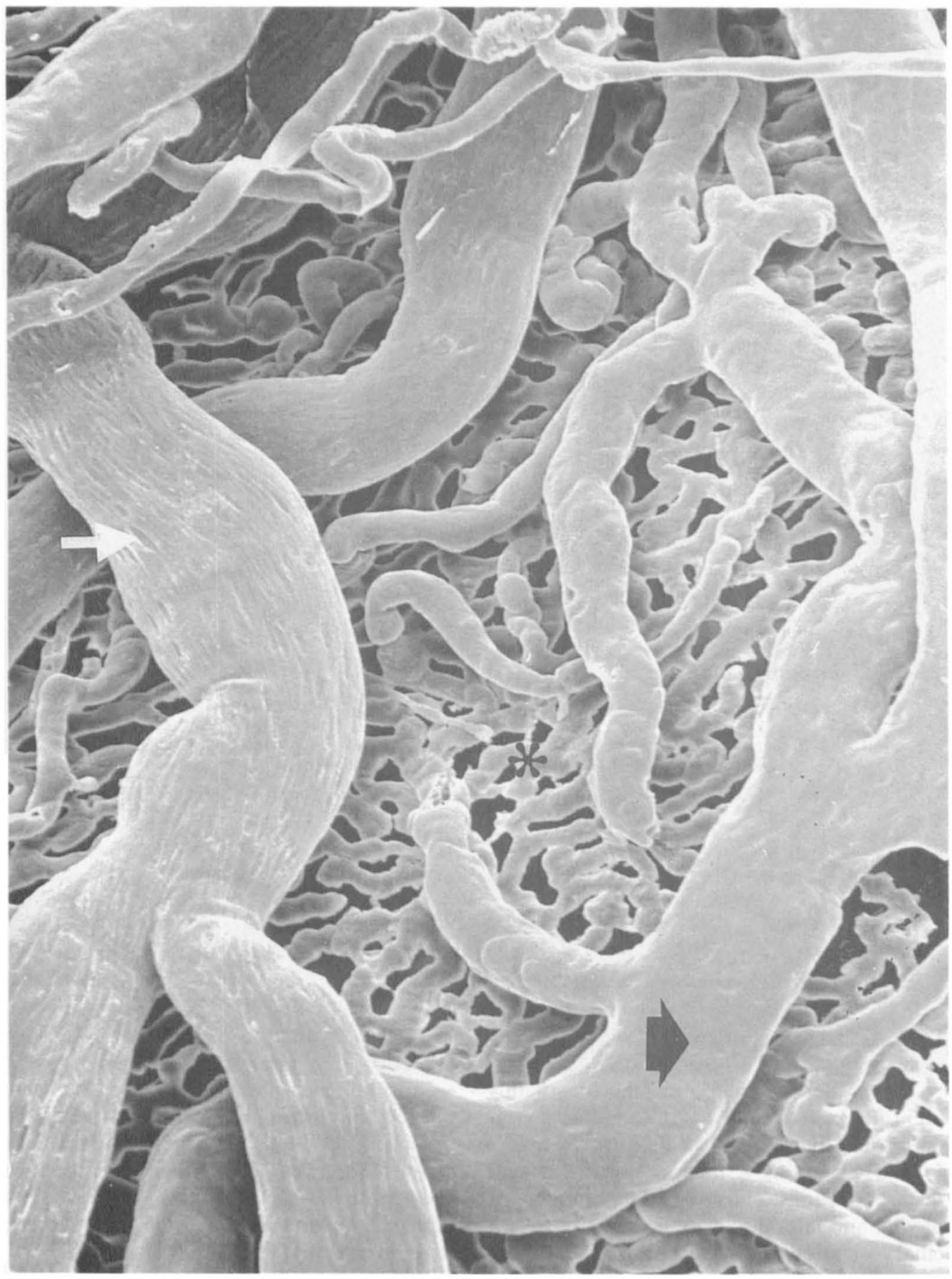

Fig. 2. Scanning electron photomicrograph of part of the choroid illustrating an artery with characteristic elongated endothelial nuclear impressions (white arrow) and contrasting vein with rounder endothelial nuclear impressions (black arrow). The choriocapillaris is in the background (star). 


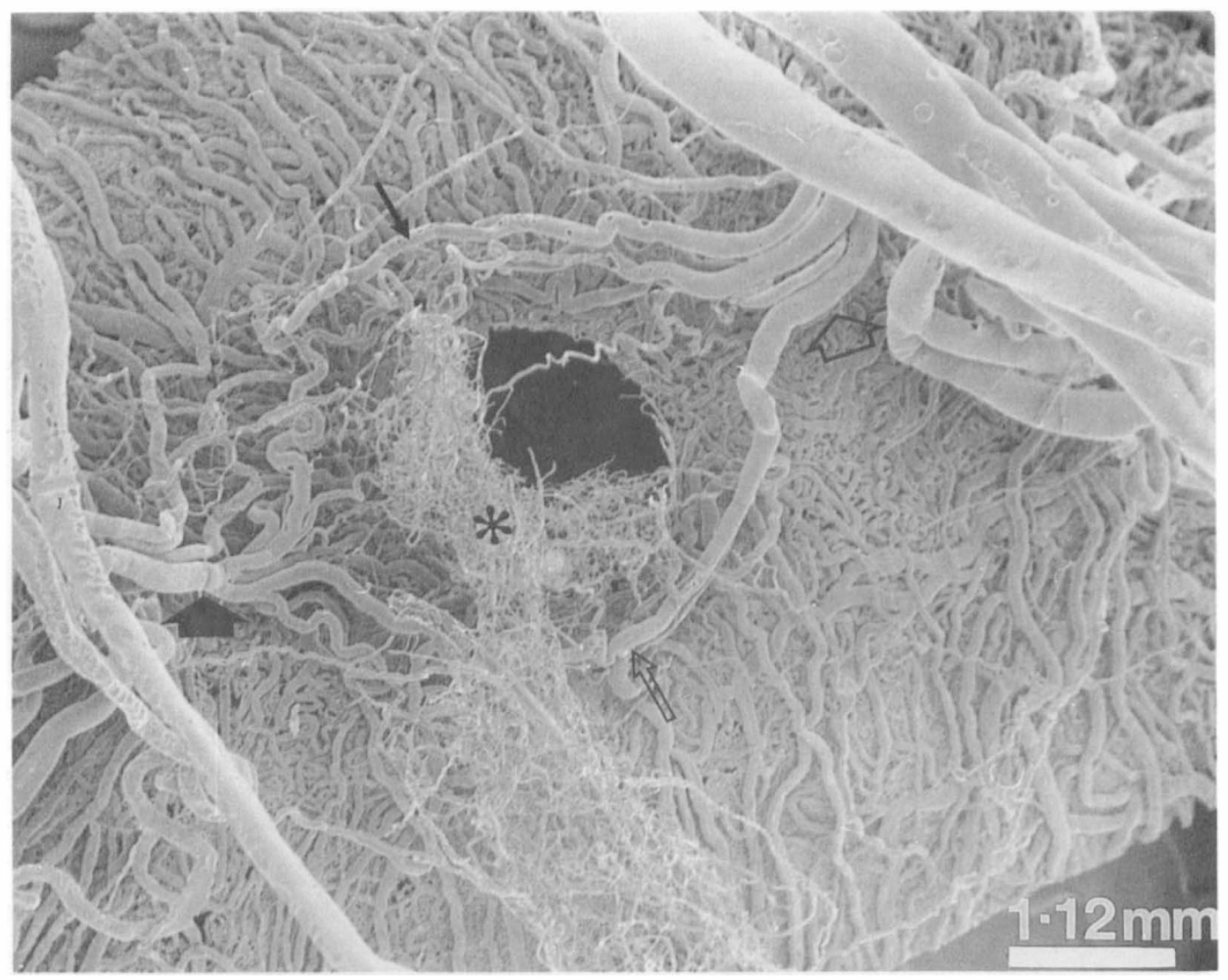

Fig. 3. Scanning electron photomicrograph of 'circle' of Haller and Zinn formed by branches of lateral short posterior ciliary arteries (short empty arrow) and a medial short posterior ciliary artery (short solid arrow) forming a superior (straight solid arrow) and inferior anastomosis (straight empty arrow). (From a right eye).

macroscopically (Fig. 1) and with scanning electron microscopy for identification of the major morphological features of the retrolaminar optic nerve vasculature. In none of the subjects was there a history of anterior ischaemic optic neuropathy. Casts of arteries and veins were easily identifiable by their characteristic endothelial nuclear impressions. (Fig. 2)

The 'circle' of Haller and Zinn was readily identifiable in all subjects in some form after dissection. It is shown in its most complete form (Fig. 3) as a microscopic, arteriolar intrascleral anastomosis between branches of medial and lateral para-optic short posterior ciliary arteries. This is a horizontal ellipse which could be divided into superior and inferior parts by the entry points of branches of a medial and lateral short posterior ciliary artery into the eye at the scleral-optic nerve junction (schematic representation Figure 5).

The microscopic intrascleral 'circle' of Haller and Zinn was distinguishable from a separate macroscopic extrascleral arterial anastomosis present superiorly in some eyes (Fig. 5). This extrascleral arterial anastomosis was formed from different (more distal) short posterior ciliary arteries.

Morphological variations can be considered in terms of form, position and branches (see Fig. 4). These variations were greatest for eyes between subjects than for eyes from the same subject (see Figs. 3, 4 same subject and Figs. 6, 7 different subject, for comparison).

\section{Form}

An arterial to arterial anastomosis between 


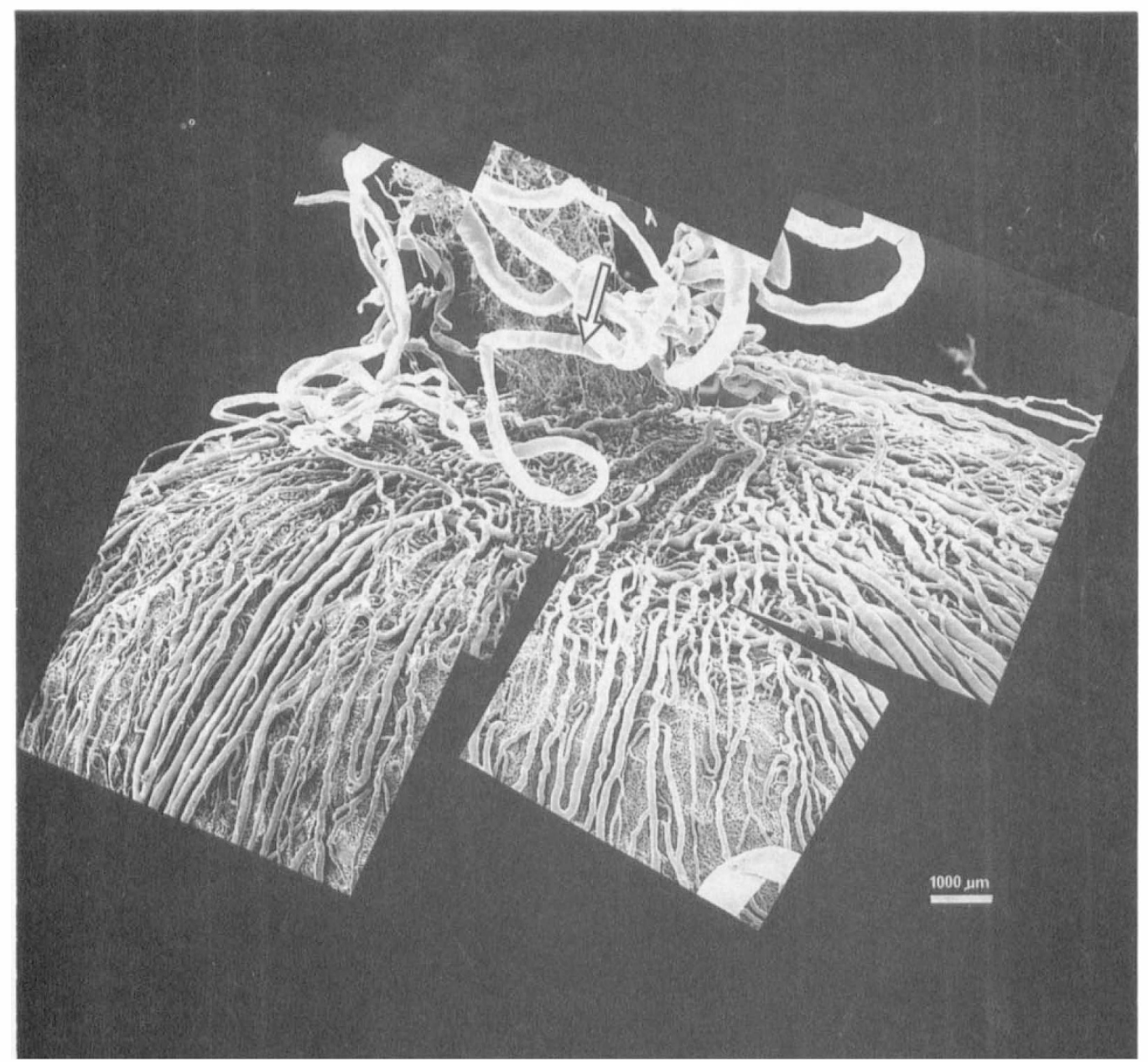

Fig. 4a. Scanning electron photomicrograph montage of a vascular cast of posterior pole of globe showing extrascleral circle (empty arrow) formed by lateral and medial short posterior ciliary arteries. (From a left eye, paired with Figure 3). 


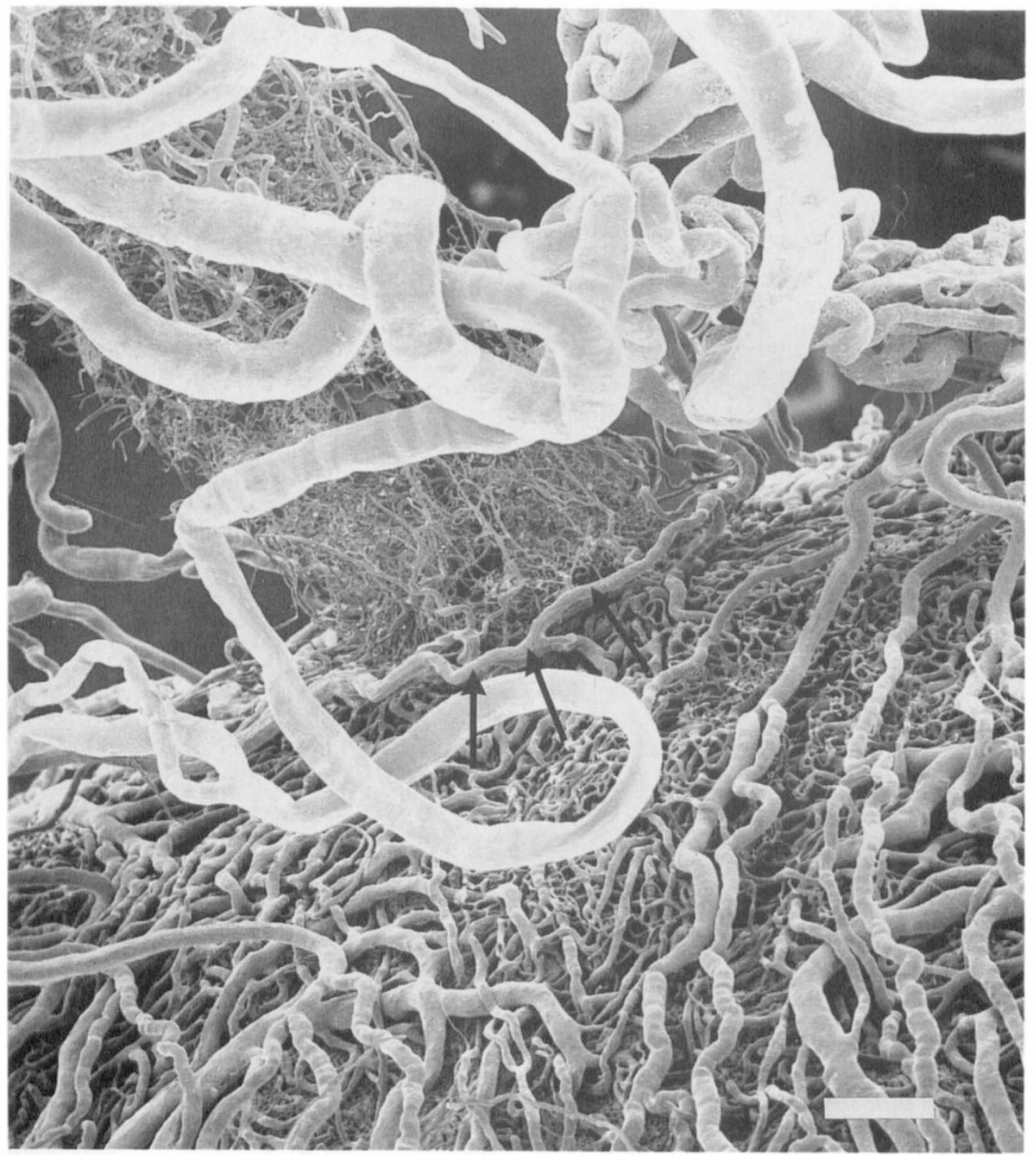

Fig. 4b. Higher magnification illustrating superior arteriolar anastomosis of 'circle' of Haller and Zinn (straight solid arrow) lying intrasclerally in this eye. Bar $=500 \mu \mathrm{m}$ 


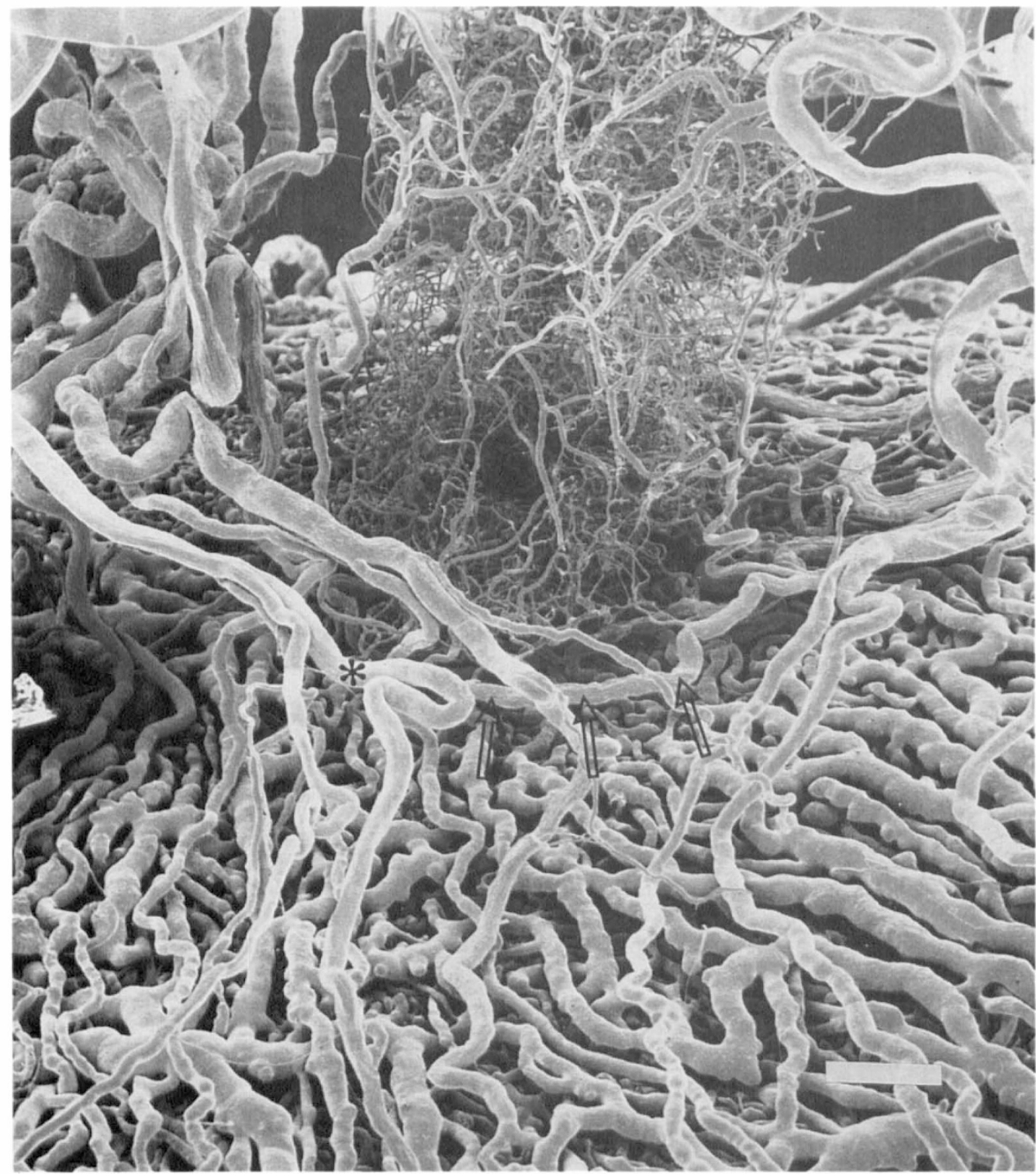

Fig. 4c. Higher magnification of inferior part of 'circle' of Haller and Zinn (empty straight arrows) from the same eye seen from the other side. A portion is obscured by a choroidal branch (star) originating from the lateral short posterior ciliary artery; continuity was confirmed by altering the tilt of the cast in the scanning electron microscope. $\mathrm{Bar}=500 \mathrm{um}$ 


\section{SCHEMATIC REPRESENTATION OF RETROLAMINAL BLOOD SUPPLY}

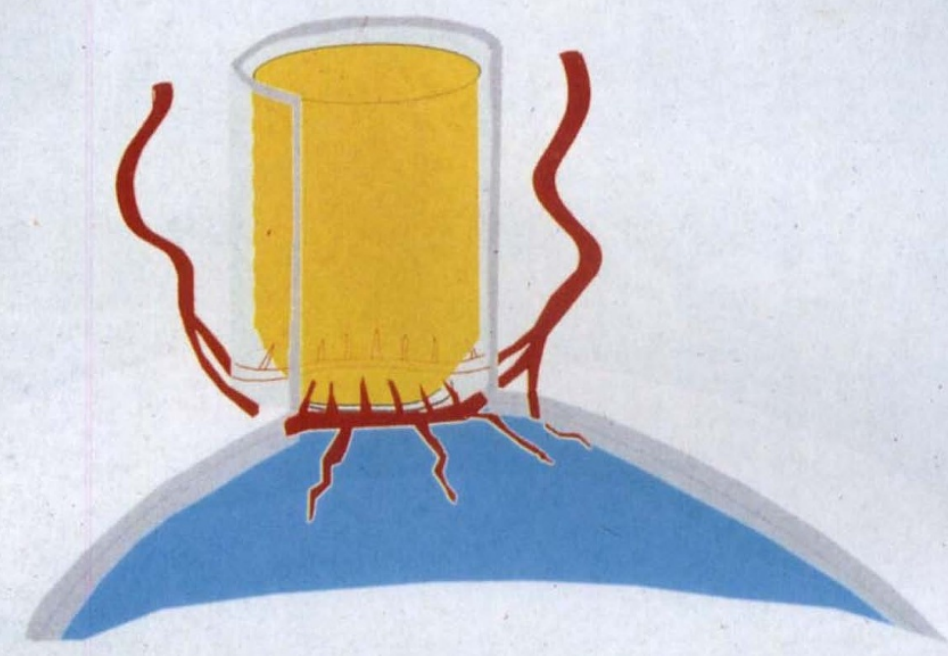

Fig. 5. Schematic representation of 'circle' of Haller and Zinn where the branches of the para-optic short posterior ciliary arteries enter the eye at the sclero-optic junction at different points dividing the ellipse horizontally into two parts. Recurrent pial and choroidal branches arise from each part.

medial and lateral short posterior ciliary arteries could be demonstrated on either the superior or inferior parts in all eyes. In some eyes the 'circle' made a complete anastomosis between medial and lateral para-optic short posterior ciliary arteries (Figs. 3, 6) with or without narrow portions (Fig. 7), or was incomplete with overlapping but not anastomosing arterioles; it could also have a double supply.

\section{Position}

The position of the 'circle' of Haller and Zinn varied in relation to the optic nerve: it could lie tightly adherent to the optic nerve (Fig. 8a) being only identifiable after extensive dissection of optic nerve capillaries or, in contrast, lie at a distance away from the optic nerve (Fig. 8b) when it was easily identifiable with minimal dissection as in Figure 3.

The axial position of the 'circle' also varied: it could be anterior, close to outer choroidal vessels where the superior and inferior parts lay intrascleral (Figs 8c, 9), or posterior, away from the choroid where the superior and inferior parts were partially or completely extrascleral (Figs. 8d, 10).

\section{Branches}

Branches derived from the 'circle' of Haller and Zinn included recurrent pial branches, recurrent choroidal branches and arteriolararteriolar anastomoses (see Figs. 11a, b). The pial branches $(30-50 \mu \mathrm{m})$ arose either independently from the 'circle' averaging four to seven arterioles from each part or as two to three trunks from each part which subsequently divided. Small branches penetrated the retrolaminar optic nerve to anastomose with optic nerve capillaries. Larger recurrent choroidal branches $(50-100 \mu \mathrm{m})$ supplied the immediate peripapillary choroid and 


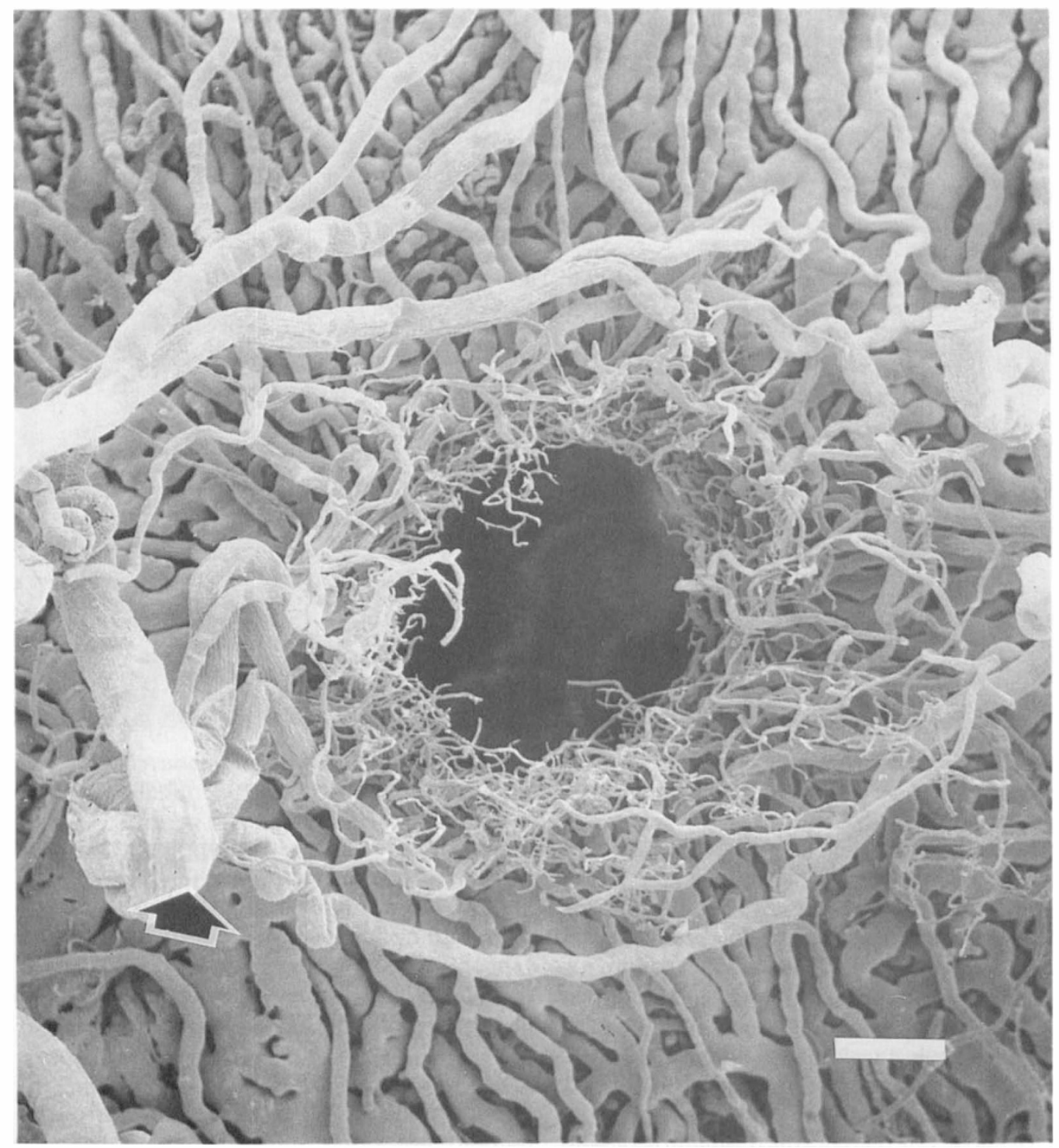

Fig. 6. Anastomoses between medial and lateral short posterior ciliary arteries forming complete 'circle' of Haller and Zinn. Note double supply temporally (solid arrow) which forms an incomplete anastomosis from which pial vessels also arise. (From a left eye). Bar $=400 \mu \mathrm{m}$. 


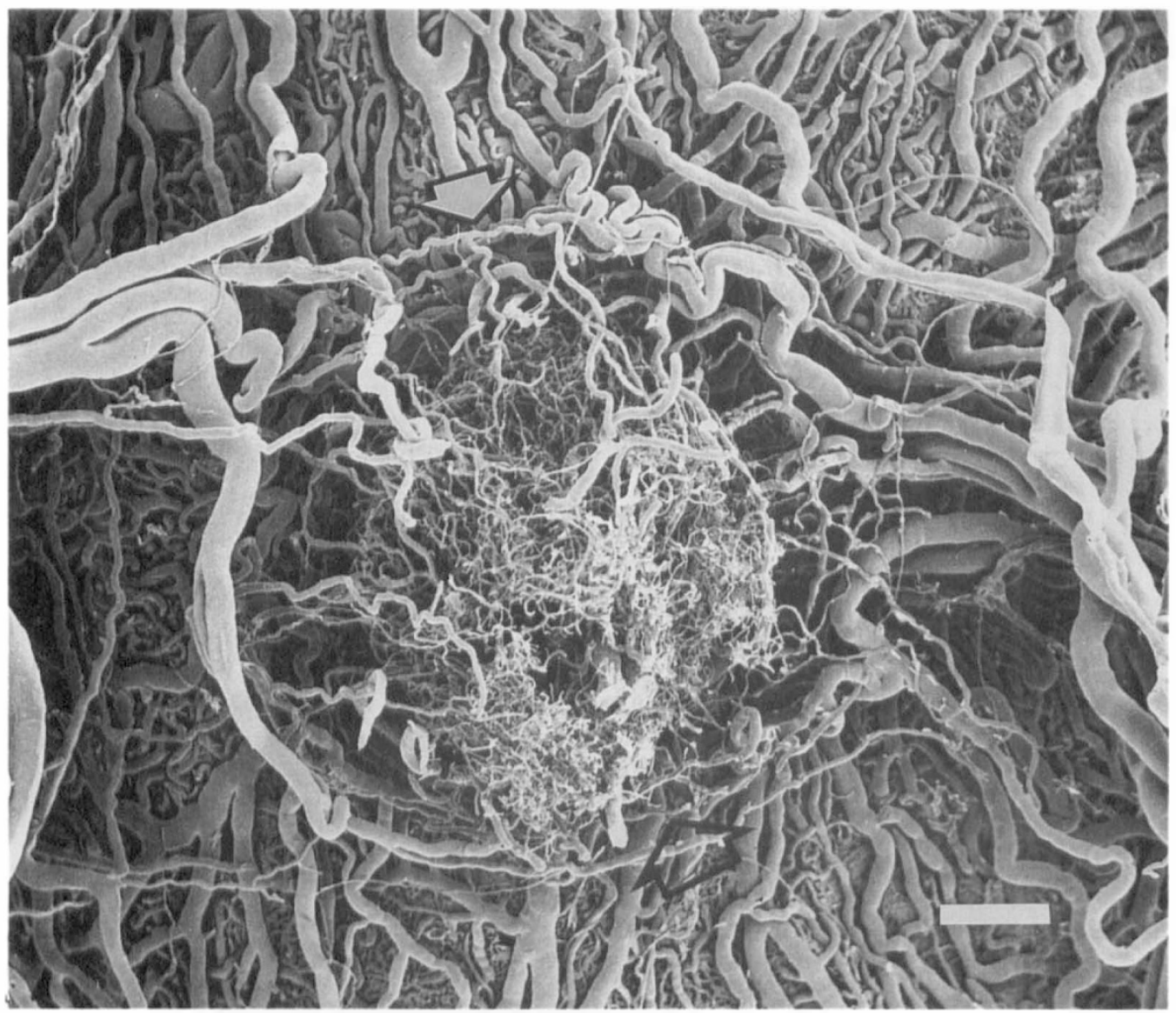

Fig. 7. Anastomoses between medial and lateral short posterior ciliary arteries forming a complete 'circle' but with sections on superior (solid arrow) and inferior (empty arrow) parts. From a left eye. bar $=500 \mu \mathrm{m}$.

extended towards the equator as straight vessels superiorly and inferiorly (Fig. 11a). Small centripetal branches $(10-30 \mu \mathrm{m})$ derived from these choroidal branches and from the choroidal edge (adjacent to the border zone of Elschnig) entered the laminar and retrolaminar optic nerve (Fig. 11b). Arteriole-arteriole anastomoses were noted within the 'circle' of Haller and Zinn and between its branches (Fig. 11a, b).

Branches of the central retinal artery were specifically studied in those casts where the optic nerve was only partially dissected. We noted branches from the central retinal artery approximately $1.5 \mathrm{~mm}$ posterior to the anterior laminar region (Fig. 12) running both centripetally and centrifugally anastomosing with optic nerve capillaries, but no branches anterior to this were seen supplying the retrolaminar optic nerve.

The blood supply of the laminar and retrolaminar optic nerve was derived from the short posterior ciliary arteries via the 'circle' of Haller and Zinn with contributions from the choroid.

\section{Discussion}

The 'circle' of Haller and Zinn is a microscopic arteriolar intrascleral anastomosis formed by branches medially and laterally of para-optic short posterior ciliary arteries. It forms a horizontal ellipse which is clearly divided into superior and inferior parts by the entry points of a medial and lateral short posterior ciliary artery into the eye (Figs. 3,4$)$. It is separate from an inconsistent macroscopic 
A.

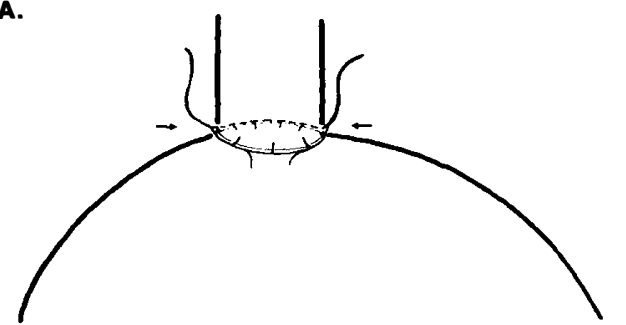

C.

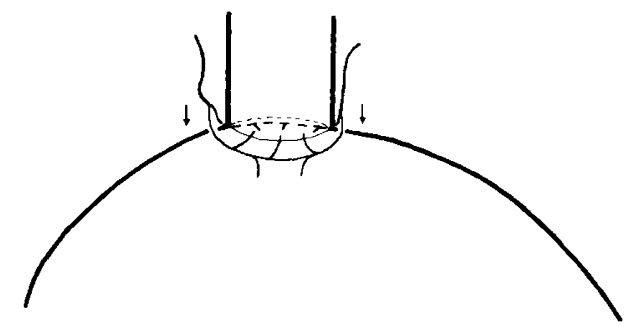

B.

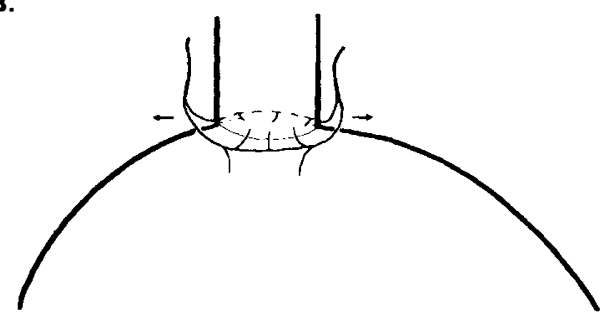

D.

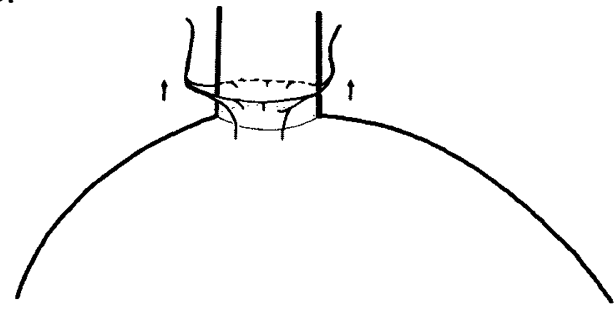

Fig. 8. Diagrams showing position of 'circle' of Haller and Zinn in relation to optic nerve. A: Tightly apposed. B: At some distance from the optic nerve. C: Anterior (intrascleral). D: Posterior (extrascleral).

extrascleral anastomosis formed by different short posterior ciliary arteries (Fig. 5). The 'circle' of Haller and Zinn supplies the retrolaminar optic nerve via its pial and recurrent choroidal branches. The posterior lamina also receives a contribution from small arterioles off the choroid (Fig. 11). Centripetal and centrifugal branches arise posterior to the retrolaminar region, a finding in accordance with other studies. ${ }^{10,11}$

The original descriptions by Haller ${ }^{12}$ and $\mathrm{Zinn}^{13}$ of an intrascleral anastomosis around the optic nerve formed by small short posterior ciliary artery branches were derived from injection and dissection of human orbital specimens (see Fig. 13). The existence of this circle has been questioned. ${ }^{6,7,14-16}$ Several authors have examined the normal blood supply of the lamina cribrosa and retrolaminar optic nerve in man from histopathological sections of injected specimens, ${ }^{7,8}$ from latex casts, ${ }^{16,18}$ and from methyl methacrylate casts, ${ }^{19}$ and report different findings. The posterior laminar and retrolaminar optic nerve was found to be supplied by branches of a complete or incomplete circle of Haller and Zinn (with a contribution from choroidal arteries), ${ }^{7,15,19}$ by branches of short posterior ciliary arteries and pial vessels in the absence of any circle,${ }^{8}$ from an inconsistent and rarely complete circle, ${ }^{16}$ in which case the retrolaminar region was supplied directly from short posterior ciliary arteries in the sclera. ${ }^{6}$ These different observations in man may be attributed to normal variation in vascular morphology and the different techniques of examination.

In sub-human primates no circle of Haller and Zinn has been found. ${ }^{14,15,17,20-22}$ It is not justifiable to use conclusions drawn from subhuman primates as directly relevant to man.

Macroscopic examination and histopathological serial sectioning are inadequate techniques to demonstrate complex threedimensional intrascleral and intraneural vascular anastomoses. Modern vascular casting techniques have overcome many of the early problems of vascular casting related to unreliable filling, extravasation and fragility and the methods used in this study produced 


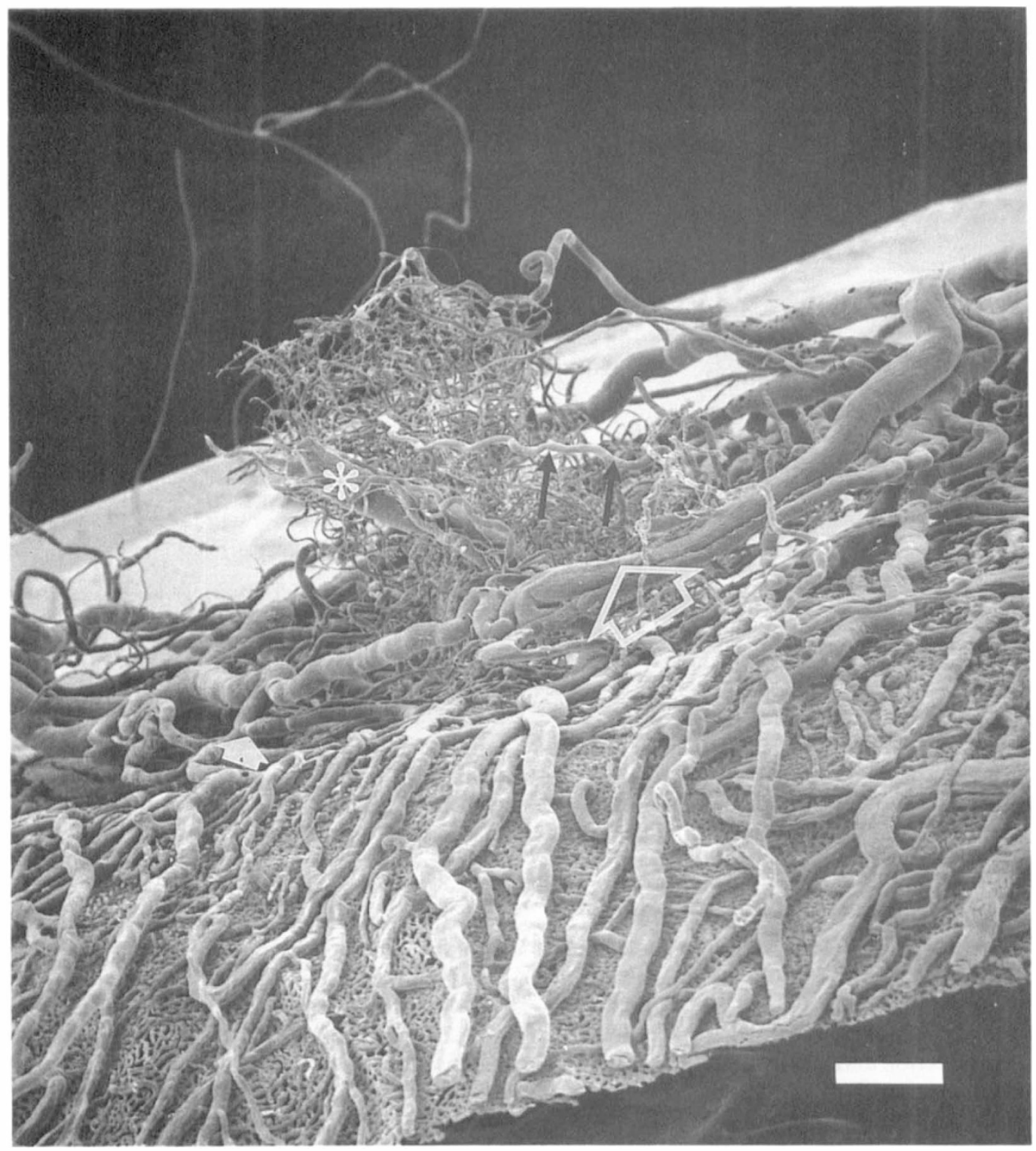

Fig. 9. Scanning electron micrograph showing axial position of 'circle' anterior (intrascleral) with inferior anastomosis (empty arrow), recurrent choroidal branches (short solid arrows) and pial branches (long arrows) and the central retinal artery (star) surrounded by residual optic nerve capillaries. From a right eye. Bar $=400 \mu \mathrm{m}$. 


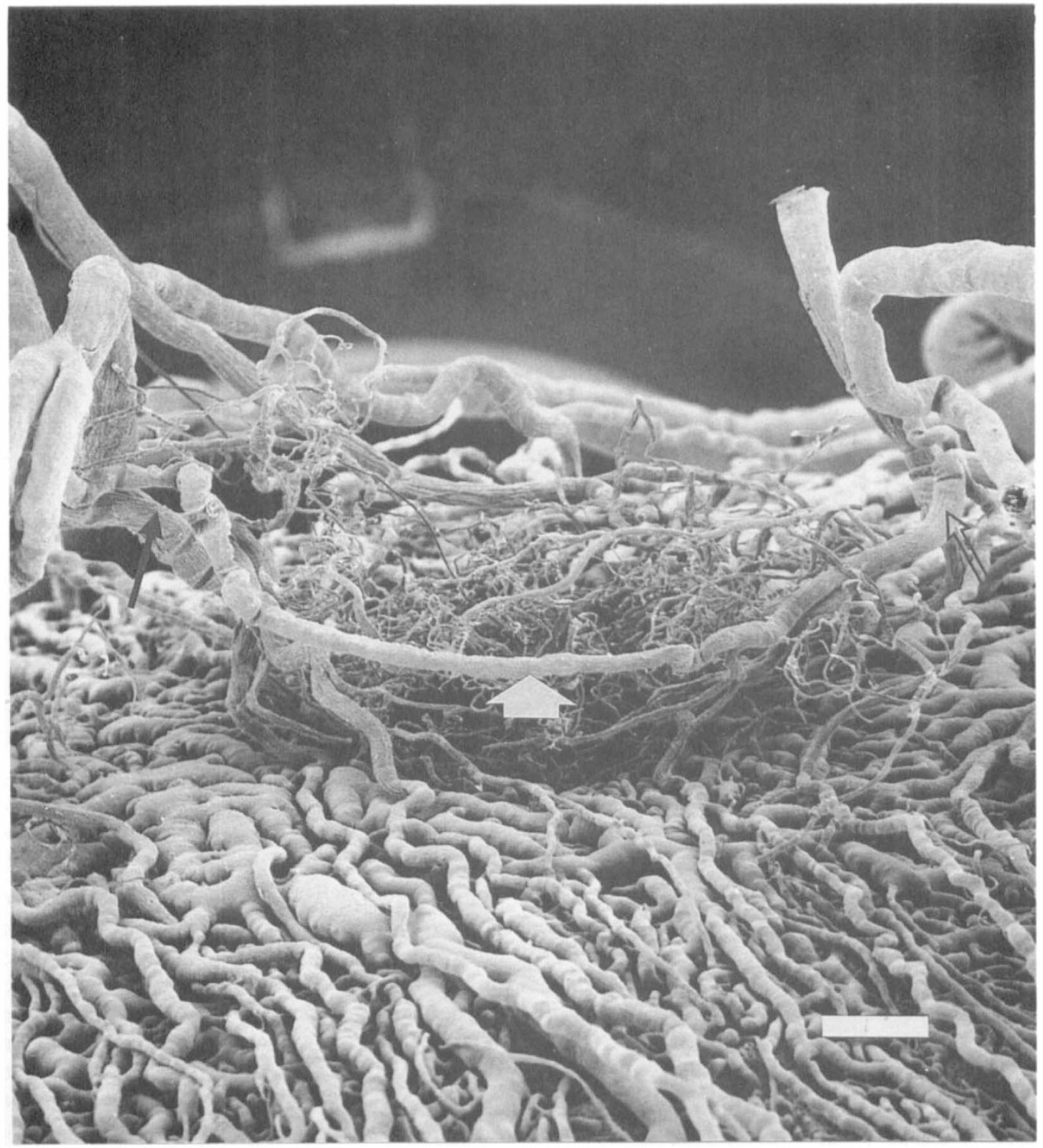

Fig. 10. Scanning electron photomicrograph showing posterior axial position of 'circle' (partially or totally extrascleral) with temporal (long solid arrow) and medial (empty arrow) short posterior ciliary artery branches form inferior anastomosis (short solid arrow). Same left eye as in figure 6. Bar $=400 \mu \mathrm{m}$. 


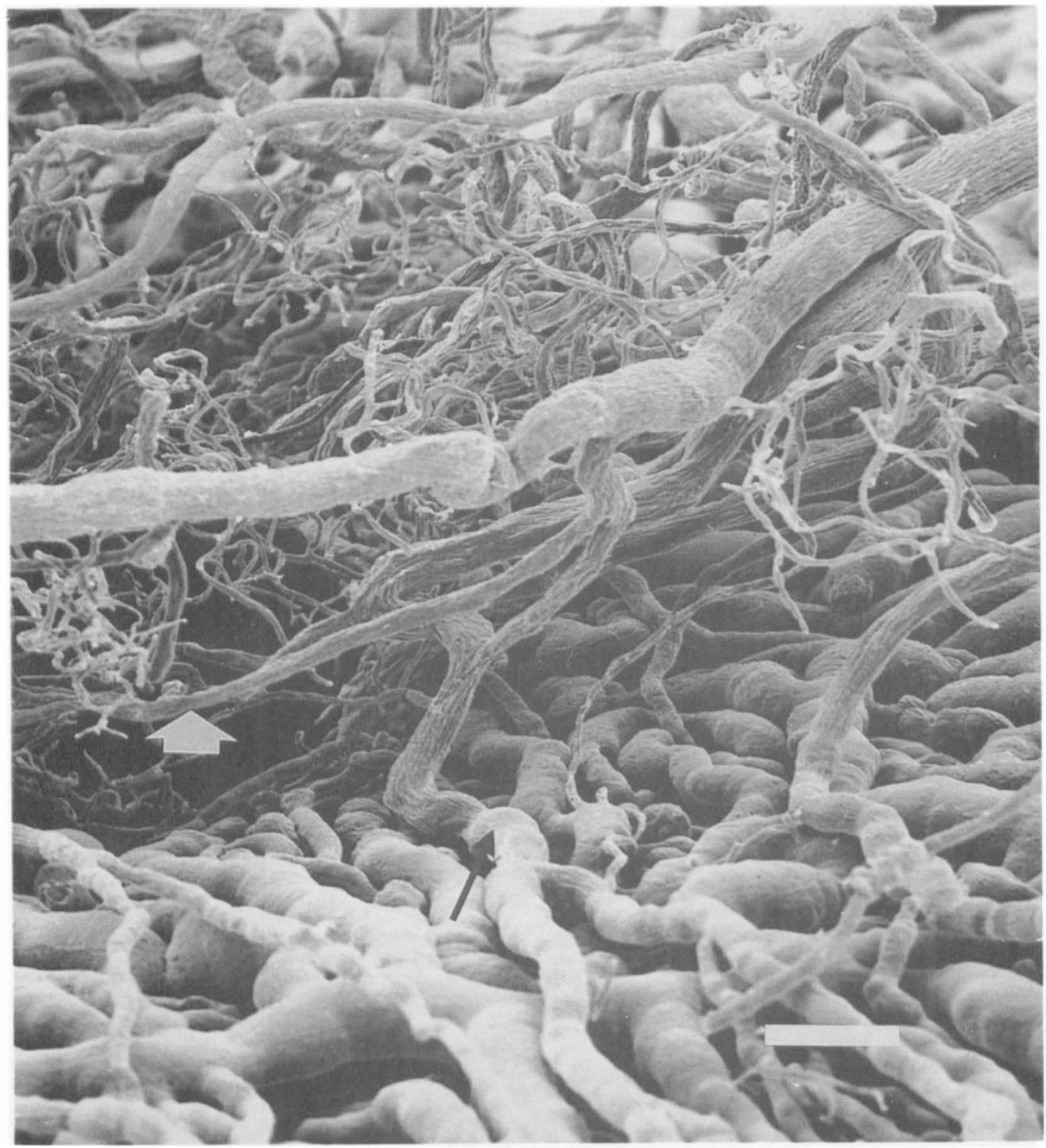

Fig. 11a. Higher magnification of medial part of inferior anastomosis seen in Figure 10 showing branches; recurrent choroidal (long solid arrow), pial vessel arising from the undersurface of the arteriole (short solid arrow) to anastomose with retrolaminar optic nerve capillaries. Arteriolar-arteriolar anastomoses are seen between the recurrent choroidal branch, the pial vessels and the 'circle'. Bar $=200 \mu \mathrm{m}$. 


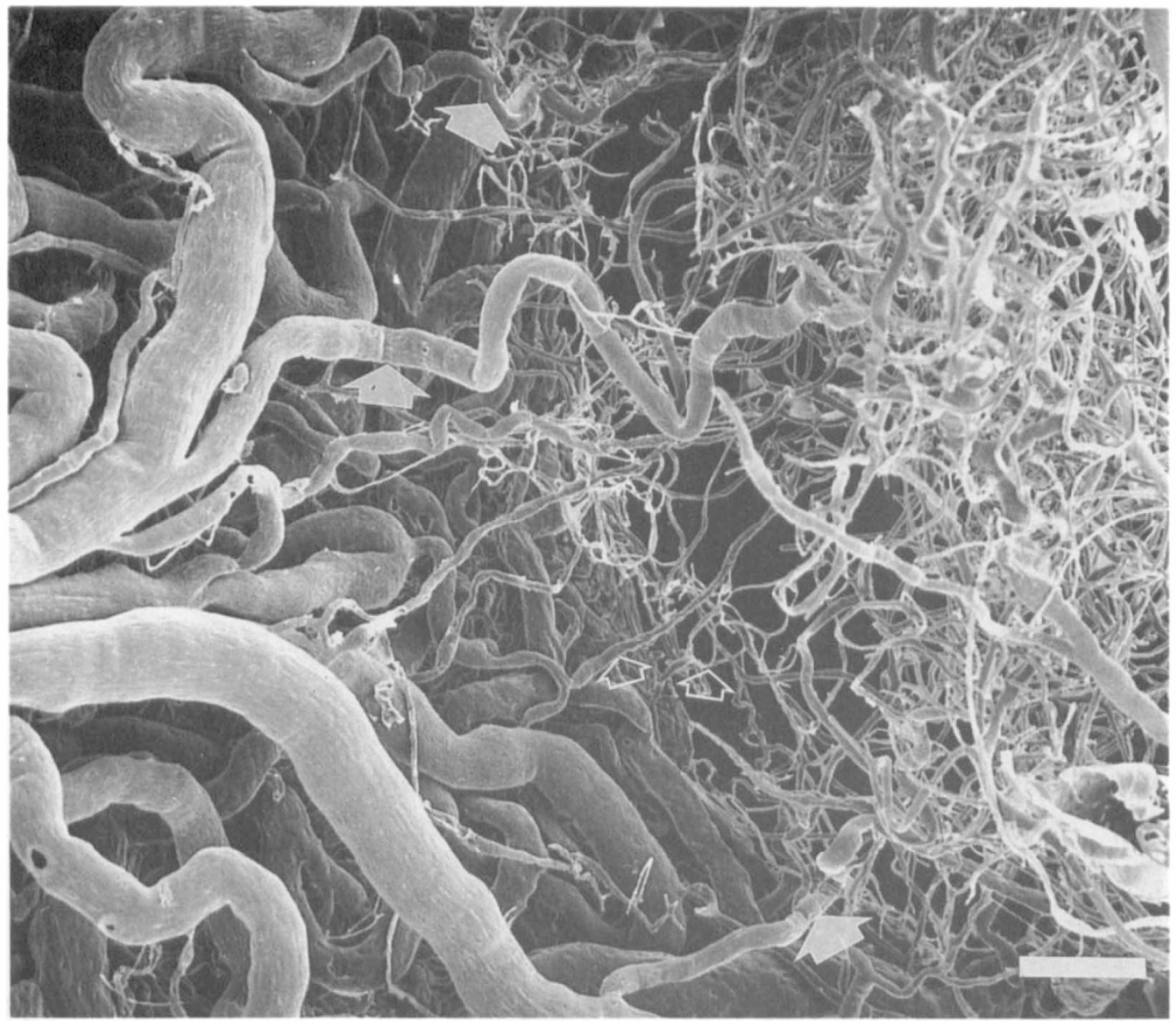

Fig. 11b. Higher magnification of medial short posterior ciliary artery branches (from the same eye shown in Figure 3) where they enter the sclera to form the superior and infeior parts of the 'circle'. Pial branches (solid arrows) and small centripetal branches both from the choroid and recurrent choroidal branches (empty arrows) enter the laminar and retrolaminar optic nerve. Same right eye as Figure 3. Bar $=200 \mu \mathrm{m}$.

reliable filling at physiological infusion pressures using low viscosity resins (in human cadaver orbits $36-48$ hours post-mortem). In this study we employed extensive and systematic dissection in a large number of methyl methacrylate casts in order to document the morphological variations of form, position and branches of the 'circle' of Haller and Zinn in man.

Our findings that the 'circle' is an ellipse with two anastomoses, superior and inferior, formed by the entry points of the short posterior ciliary artery branches, not only clarifies the vasculature in this region but supplies an anatomical basis for clinical pathology. Vascular casting does not directly tell us about functional blood flow, but provides a foun- dation on which our understanding of function is based.

Structural factors are believed to be important in the pathogenesis of non-arteritic anterior ischaemic optic neuropathy ${ }^{23-27}$ The purpose of studying the normal vascular anatomy of the retrolaminar region was to relate these anatomical findings to clinical observations in non-arteritic anterior ischaemic optic neuropathy, namely the altitudinal pattern of visual loss, affecting the inferior-nasal field preferentially. ${ }^{1-3}$

If the retrolaminar blood supply is from pial branches of the medial and lateral short posterior ciliary arteries and from small recurrent branches from the choroid, ${ }^{6-8}$ it is perfectly justifiable to postulate watershed infarcts ver- 


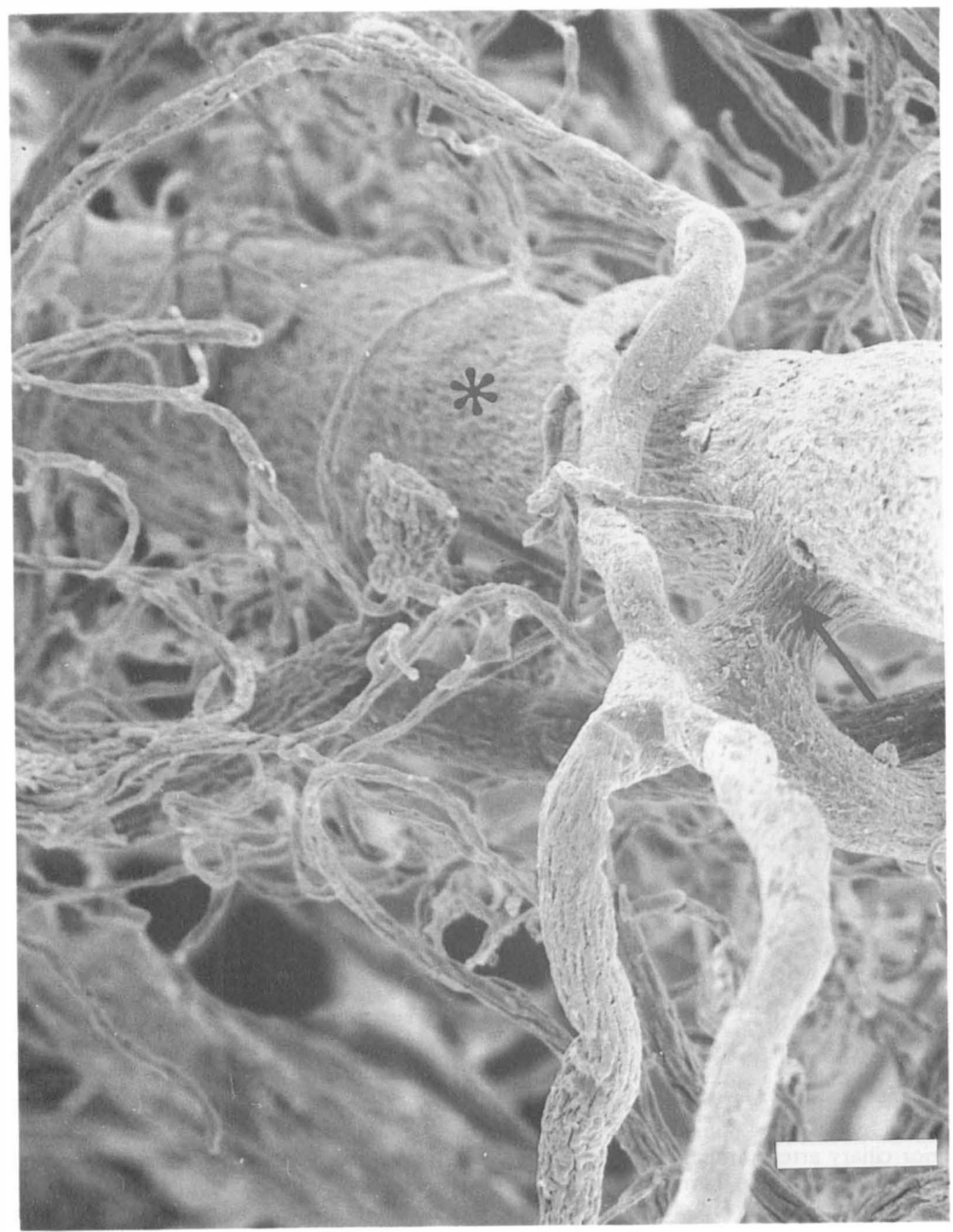

Fig. 12. Branch (arrow) from intraneural central retinal artery (flower) arising approximately $1.5 \mathrm{~mm}$ posterior to lamina to anastomose with optic nerve capillaries. Bar $=125 \mu \mathrm{m}$. 


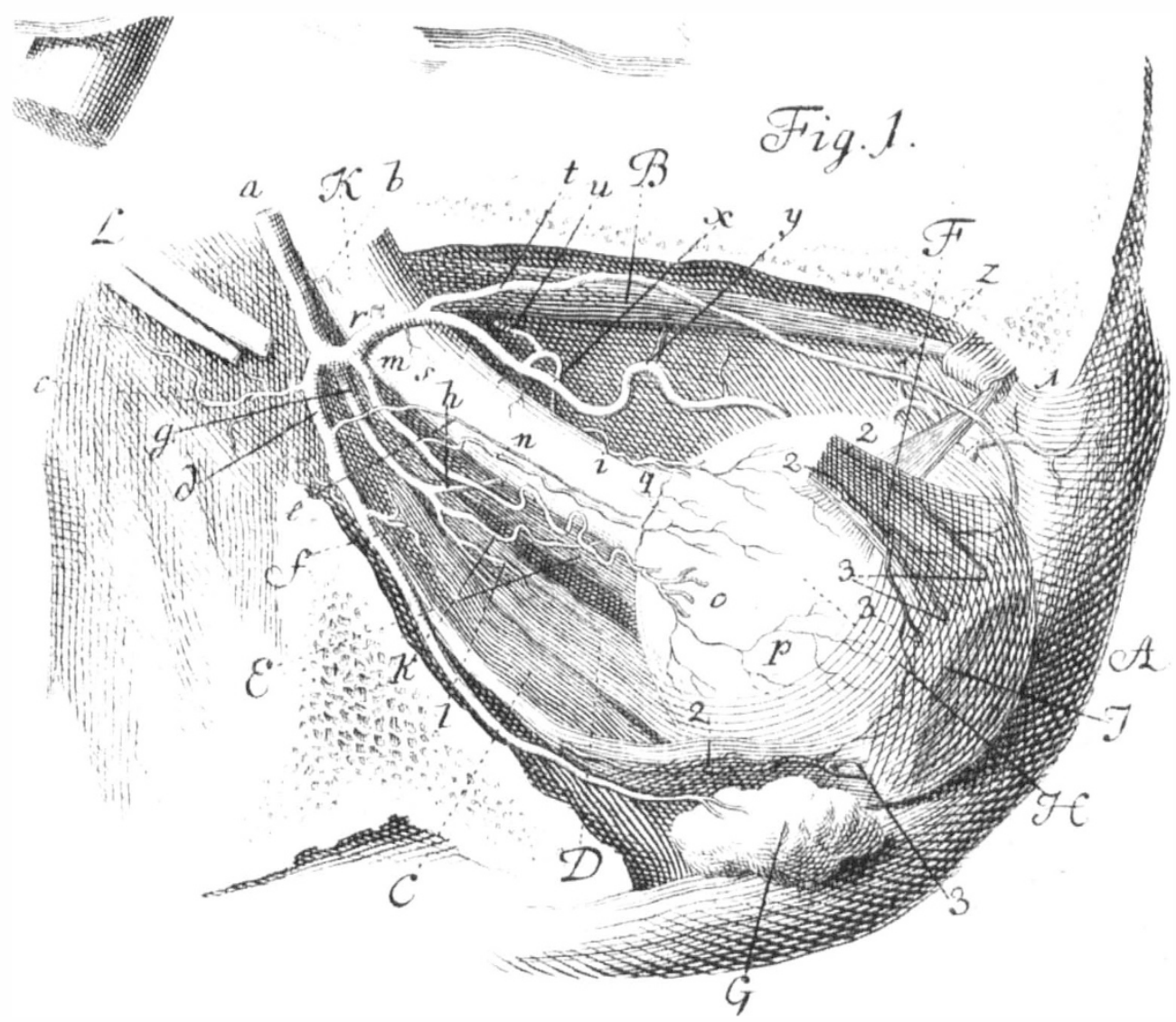

Fig. 13. Zinn's original woodcut of the blood supply of the eye based on dissections showing the position of the 'circle' of Haller and Zinn at (q). Made in 1755.

tically at 12 and 6 o'clock. This pattern is not seen in clinical practice. We have shown that there are pial vessels coming off the 'circle' of Haller and Zinn from above and below providing an altitudinal blood supply to the retrolaminar optic nerve. A reduction of perfusion pressure in this circulation would result in selective infarction and explain the altitudinal pattern of visual field loss seen in non-arteritic anterior ischaemic optic neuropathy if there was a preferential vascular supply to the inferior optic nerve. These findings do not provide an anatomic explanation for the preferential inferior field loss; further quantitative studies are in progress to see if there is a preferential anastomotic blood supply to the inferior retrolaminar optic nerve.

We are grateful to Robin Howes for technical assistance and to Professor Robert Weale for translating from the Latin the relevant sections on the blood supply of the retrolaminar optic nerve from the book by Zinn.

Miss Olver is supported by the Francis and Renee Hock Trust.

\section{References}

${ }^{1}$ Boghen DR and Glaser JS: Ischaemic optic neuropathy. The clinical profile and natural history. Brain 1975, 98: 689-708.

${ }^{2}$ Hayreh SS: Anterior ischaemic optic neuropathy. 1. Terminology and pathogenesis. Br J Ophthalmol 1974, 58: 955-63.

${ }^{3}$ Hayreh SS and Podhajsky P: Visual field defects in anterior ischaemic optic neuropathy. Doc Ophthalmol Proc Ser 1979, 19: 53-71.

${ }^{4}$ Henkind P, Charles NG, Pearson J: Histopathology of ischaemic optic neuropathy. Am J Ophthalmol 1970, 69: 78-90. 
${ }^{5}$ Cogan DG: Neurology of the visual system. Illinois: Charles C Thomas, 1968: 185-8.

${ }^{6}$ Hayreh SS: Blood supply and vascular disorders of the optic nerve. Anal Inst Barrquer 1963, 4: 4.

${ }^{7}$ Levitzky $M$ and Henkind P: Angioarchitecture of the optic nerve. II Lamina cribrosa. Am J Ophthalmol 1969, 68: 986-96.

${ }^{8}$ Lieberman MF, Maumenee AE, Green WR: Histologic studies of the vasculature of the anterior optic nerve. Am J Ophthalmol 1976, 82: 405-23.

${ }^{9}$ Olver JM and McCartney ACE: Orbital and ocular microvascular corrosion casting in man. Eye 1989, 3: 588-96.

${ }^{10}$ Singh S and Dass R: The central artery of the retina. II A study of its distribution and anastomoses. $\mathrm{Br}$ J Ophthalmol 1960, 44: 280-99.

${ }^{11}$ Hayreh SS: The central artery of the retina. Its role in the blood supply of the optic nerve. $\mathrm{Br} J \mathrm{Oph}$ thalmol 1963, 47: 651-63.

${ }^{12}$ Haller A: Arteriarum oculi historia et tabulae arteriarum oculi. Gottingen, 1754. Cited by Francois et al in Br J Ophthalmol 1954, 38: 472-88.

${ }^{13}$ Zinn IG: Descriptio anatomica oculi humani. 1st ed. Gottingen. Abrami Vandenhoeck, 1755: 216-7.

${ }^{14}$ Anderson DR: Vascular supply to the optic nerve of primates. Am J Ophthalmol 1970, 70: 341-51.

${ }^{15}$ Anderson DR and Braverman S: Reevaluation of the optic disc vasculature. Am J Ophthalmol 1976, 82: 165-74.

${ }^{16}$ Hayreh SS: Anterior ischaemic optic neuropathy. Springer-Verlag. Berlin Heidelberg New York 1975.

${ }^{17}$ Hayreh SS: The orbital vessels of rhesus monkey. Exp Eye Res 1964, 3: 16-30.
${ }^{18}$ Hayreh SS: Blood supply of the optic nerve head and its role in optic atrophy, glaucoma, and oedema of the optic disc. Br J Ophthalmol 1969, 53: 721-48.

${ }^{19}$ Fryczkowski AW, Grimson BS, Peiffer RL: Scanning electron microscopy of vascular casts of the human scleral lamina cribrosa. Int Ophthalmol 1984, 7: 95-100.

${ }^{20}$ Shimizu K: Structure of orbital vessels. Igaku-Shoin Ltd, Tokyo 1978 p 108-124.

${ }^{21}$ Risco JM, Grimson BS, Johnson PT: Angioarchitecture of the ciliary artery circulation of the posterior pole. Arch Ophthalmol 1981, 99: 865-8.

${ }^{22}$ Ojima M: Studies on the angioarchitecture of the optic nerve. 1. Relation to the ciliary arterial circulation. Acta Soc Ophthalmol Jpn 1977, 81: 642-49.

${ }^{23}$ Beck RW, Savino PJ, Repka MX, Schatz NJ, Sergott $\mathrm{RC}$ : Optic disc structure in anterior ischaemic optic neuropathy. Ophthalmol 1984, 91: 1334-7.

${ }^{24}$ Beck RW, Servais GE, Hayreh SS: Anterior ischaemic optic neuropathy. IX. Cup-to-disc ratio and its role in pathogenesis Ophthalmol 1987, 94: 1503-8.

${ }^{25}$ Repka MX, Savino PJ, Schatz NJ, Sergott RC: Clinical profile and long-term implications of anterior ischaemic optic neuropathy. Am J Ophthalmol 1983, 96: 478-83.

${ }^{26}$ Borchert $\mathrm{M}$ and Lessell S: Progressive and recurrent non-arteritic anterior ischaemic optic neuropathy. Am J Ophthalmol 1988, 106: 443-9.

${ }^{27}$ Kline LB: Progression of visual defects in ischaemic optic neuropathy. Am J Ophthalmol 1988, 106: 199-203. 